\title{
Intermittent hypoxia alters gut microbiota diversity in a mouse model of sleep apnoea
}

\author{
Isabel Moreno-Indias ${ }^{1,2,9}$, Marta Torres ${ }^{3,4,9}$, Josep M. Montserrat ${ }^{3,4,5}$, \\ Lidia Sanchez-Alcoholado ${ }^{1,2}$, Fernando Cardona ${ }^{1,2}$, Francisco J. Tinahones ${ }^{1,2}$, \\ David Gozal ${ }^{6}$, Valeryi A. Poroyko6, Daniel Navajas ${ }^{4,7,8}$, Maria I. Queipo-Ortuño ${ }^{1,2}$ \\ and Ramon Farré $4,5,7$
}

Affiliations: 'Unidad de Gestión Clínica de Endocrinología y Nutrición, Instituto de Investigación Biomédica de Málaga (IBIMA), Malaga, Spain. ${ }^{2}$ Centro de Investigación Biomédica en Red de Fisiopatología de la Obesidad y la Nutrición (CIBER), Madrid, Spain. ${ }^{3}$ Laboratori del Son, Hospital Clínic, Universitat de Barcelona, Barcelona, Spain. ${ }^{4}$ Centro de Investigación Biomédica en Red de Enfermedades Respiratorias, CIBER, Madrid, Spain. ${ }^{5}$ Institut Investigacions Biomediques August Pi Sunyer, Barcelona, Spain. ${ }^{6}$ Section of Pediatric Sleep Medicine, Dept of Pediatrics, Pritzker School of Medicine, The University of Chicago, Chicago, IL, USA. ${ }^{7}$ Unitat de Biofísica i Bioenginyeria, Facultat de Medicina, Universitat de Barcelona-IDIBAPS, Barcelona, Spain. ${ }^{8}$ Institut Bioenginyeria de Catalunya, Barcelona, Spain. ${ }^{9}$ These authors contributed equally.

Correspondence: María I. Queipo Ortuño, Laboratorio de Investigación Biomédica $1^{a}$ planta, Instituto de Investigación Biomédica (IBIMA), Complejo Hospitalario de Málaga (Virgen de la Victoria), Campus de Teatinos s/n 29010-Malaga, Spain. E-mail: maribelqodgmail.com

ABSTRACT We assessed whether intermittent hypoxia, which emulates one of the hallmarks of obstructive sleep apnoea (OSA), leads to altered faecal microbiome in a murine model.

In vivo partial pressure of oxygen was measured in colonic faeces during intermittent hypoxia in four anesthetised mice. 10 mice were subjected to a pattern of chronic intermittent hypoxia $\left(20 \mathrm{~s}\right.$ at $5 \% \mathrm{O}_{2}$ and $40 \mathrm{~s}$ at room air for $6 \mathrm{~h} \cdot \mathrm{day}^{-1}$ ) for 6 weeks and 10 mice served as normoxic controls. Faecal samples were obtained and microbiome composition was determined by $16 \mathrm{~S}$ rRNA pyrosequencing and bioinformatic analysis by Quantitative Insights into Microbial Ecology.

Intermittent hypoxia exposures translated into hypoxia/re-oxygenation patterns in the faeces proximal to the bowel epithelium $(<200 \mu \mathrm{m})$. A significant effect of intermittent hypoxia on global microbial community structure was found. Intermittent hypoxia increased the $\alpha$-diversity (Shannon index, $\mathrm{p}<0.05$ ) and induced a change in the gut microbiota (ANOSIM analysis of $\beta$-diversity, $\mathrm{p}<0.05$ ). Specifically, intermittent hypoxia-exposed mice showed a higher abundance of Firmicutes and a smaller abundance of Bacteroidetes and Proteobacteria phyla than controls.

Faecal microbiota composition and diversity are altered as a result of intermittent hypoxia realistically mimicking OSA, suggesting the possibility that physiological interplays between host and gut microbiota could be deregulated in OSA.

@ERSpublications

Faecal microbiota composition and diversity are altered due to intermittent hypoxia mimicking OSA in a murine model http://ow.ly/ERjA9

Support statement: This work was supported, in part, by the Spanish Ministry of Economy and Competitiveness (SAF2011-22576). The research group belongs to the Centros de Investigación en Red (CIBER, CB06/03/0018) of the Instituto de Salud Carlos III (Madrid, Spain). I. Moreno Indias was supported by a Sara Borrell Postdoctoral contract (CD12/00530). M.I. Queipo-Ortuño acknowledges support from the Miguel Servet Type I programme (CP13/00065) and F. Cardona acknowledges support from the Miguel Servet Type II programme (CP13/ 00023) from the Instituto de Salud Carlos III.

Conflict of interest: None declared.

Copyright @ERS 2015 


\section{Introduction}

Obstructive sleep apnoea (OSA) has emerged as a very relevant public health problem because of its high prevalence (e.g. 10\% in males aged 30-49 years) [1], but more importantly because, in addition to reduction in quality of life and increase in traffic accidents, OSA imposes important mid- and long-term consequences, namely cardiovascular, metabolic, cognitive and cancer-related alterations. Remarkably, it is anticipated that the number of patients suffering from OSA worldwide will increase further given the close association between being overweight/obesity and OSA [2], and the evolving trends in the obesity epidemic in both developed and developing countries. Among the physiological insults experienced by patients with OSA, disruption of sleep architecture, increased sympathetic activation and intermittent hypoxia, the latter seems to play a major role since it triggers inflammation and oxidative stress cascades that are deleterious and contribute to the multi-organ morbid consequences of OSA [3].

Whereas the magnitude and pathophysiological impact of hypoxia/re-oxygenation has been investigated in different organs and tissues such as brain, liver, testes, fat or muscle [4-6], no attention has been paid to the potential effect of recurrent oxygen desaturations that characterise OSA on the gut microbiota. In fact, the mammalian gut is populated by a complex and dense microbial community dominated by obligate anaerobic organisms from both the Firmicutes and Bacteroidetes phyla in a dynamic environment determined by the host physiology [7]. In addition, various lines of evidence have also implicated both aerobic and facultative anaerobic bacteria present in the intestine in the dynamic configuration and stability of the anaerobic environment inside the gut [8,9]. Alterations in gut microbiota induced by episodic changes in blood oxygen content could be relevant since metabolic alterations, such as obesity and metabolic syndrome, which are modulated by the gut microbiome, are commonly associated with OSA $[10,11]$. Indeed, most OSA patients are obese and in approximately half of patients the metabolic syndrome is concurrently present [12]. Interestingly, new available data also support the notion that OSA per se may feed back into mechanisms resulting in the development or reinforcement of obesity [13].

With the aim to generate initial evidence shedding light onto this unknown aspect of OSA, we hypothesised that the intermittent arterial hypoxaemia characterising OSA will result in hypoxia/ re-oxygenation cycling events within the gut microbiome and that, as a result, the biological diversity of gut microorganisms may be modified. The rationale of this hypothesis is based on the evidence that the wall of blood capillaries in the epithelial surface of the bowel, which are very permeable because of their main function of absorbing nutrients from the gut content in food digestion, obviously allows for oxygen diffusion. In fact, it is well known that although the core of the gut content is anoxic, there is a gradient in the oxygen concentration of the microbiome within a $\approx 150-200 \mu \mathrm{m}$ range, in the vicinity of the gut epithelium [8]. Accordingly, a recent study of the intestinal microbiota of mice and humans reported the existence of a radial gradient of microbes linked to the distribution of oxygen and nutrients provided by the host tissue. In this context, hyperbaric oxygen treatment altered the composition of the gut microbiota in mice. In humans, $16 \mathrm{~S}$ rRNA gene analyses revealed an increased proportion of oxygen-tolerant organisms of the Proteobacteria and Actinobacteria phyla associated with the rectal mucosa, compared with the faeces, indicating an effect of oxygen concentrations on the microbiota [9]. Thus, it is plausible to postulate that, as a result of diffusion, the intermittent changes in oxygen content in the capillary blood associated with OSA induce fluctuations in oxygen levels in the microbiome.

To test our hypothesis, we carried out a study on mice exposed to a conventional model of chronic intermittent hypoxia mimicking OSA. First, we measured partial pressure of oxygen within the faeces to confirm that intermittent arterial hypoxaemia translates into hypoxia/re-oxygenation events within the microbiome. Secondly, we carried out a taxonomic analysis of faecal microbiota in animals subjected to chronic intermittent hypoxia and in normoxic control mice to analyse potential microbiome alterations in the gut.

\section{Material and methods}

Animals

The study was carried out on 24 pathogen-free C57BL/6 male mice aged 6 weeks (Charles River Laboratories, Saint Germain sur L'arbresle, France). 10 days before starting the study and during the 6-week study duration animals were housed in standard cages and received tap water and sterilised standard food ad libitum. They were kept in a temperature and light controlled room in the animal facilities. The experimental procedures were approved by the Ethical Committee for Animal Research of the University of Barcelona (Barcelona, Spain).

In vivo assessment of partial pressure of oxygen inside the gut

The first series of experiments focused on evaluating whether breathing intermittent hypoxic gas mixtures, which mimic the inspired fractions of oxygen leading to the typical oxyhaemoglobin desaturations that 
occur in OSA, will actually translate into hypoxia-/re-oxygenation-induced changes at the microbiota level. Due to this, partial pressure of oxygen $\left(\mathrm{PaO}_{2}\right)$ in the small bowel faeces was measured in four anesthetised (urethane $20 \%, 1 \mathrm{~g} \cdot \mathrm{kg}^{-1}$ ) mice whose arterial oxygen saturation was measured by pulse oximetry (MouseOx Plus; Starr Life Sciences Corp., Oakmont, PA, USA). After anaesthesia, the abdominal wall was opened and the intestines were exposed. A minimal incision was made in the small bowel wall to insert a fast-response Clark's polarographic oxygen microelectrode pipette (OX-50; Unisense A/S, Aarhus, Denmark) $(50 \mu \mathrm{m}$ diameter, $90 \%$ response time $<2 \mathrm{~s})$, which was connected to an amplified picoammeter (Unisense A/S) previously calibrated in water at $100 \% \mathrm{O}_{2}, 21 \% \mathrm{O}_{2}$ and oxygen-free solution $(\mathrm{NaOH}$ $0.1 \mathrm{M}$, sodium ascorbate $0.1 \mathrm{M}$ ) and oxygen pressure was recorded (MicOX software; Unisense A/S). The tip of the microelectrode sensor was progressively advanced by means of a microcontrolled drive system through the faecal content of the small intestine until it reached the bowel epithelium at the opposite side from where the sensor was inserted. Then, intermittent hypoxia (room air: $40 \mathrm{~s} ; 5 \% \mathrm{O}_{2}$ air: $20 \mathrm{~s}$ ) was administered to the mice via a sealed nasal mask. Subsequently, the microelectrode was progressively retrieved to record oxygen pressure at different distances from the bowel epithelium.

\section{Application of chronic intermittent hypoxia}

For intermittent hypoxia exposure, 10 mice were weighed and placed in a box $(26 \mathrm{~cm}$ long, $18 \mathrm{~cm}$ wide, $6 \mathrm{~cm}$ high) flushed with air with cyclic changes in oxygen content (40 s of room air and $20 \mathrm{~s}$ of hypoxic air at $5 \% \mathrm{O}_{2}$ ) as described in detail elsewhere [6]. This intermittent hypoxia, which mimics a rate of 60 apnoeas $\cdot \mathrm{h}^{-1}$ typical of severe OSA, was applied for $6 \mathrm{~h} \cdot$ day $^{-1}(09: 00-15: 00)$ for 6 weeks. 10 mice were weighed and placed in a similar box continuously flushed with room air (normoxic controls). After the 6-week application of intermittent hypoxia (or normoxia), the mice were weighed and faecal samples were obtained directly from stool expulsion stimulated by manual handling of the animal, and were immediately frozen at $-80^{\circ} \mathrm{C}$ and stored until analysed. At the end of the study, the animals were anesthetised (urethane $20 \%, 1 \mathrm{~g} \cdot \mathrm{kg}^{-1}$ ) and euthanised by exsanguination through the abdominal aorta.

\section{DNA extraction from faecal samples}

DNA extraction from $200 \mathrm{mg}$ of faecal material was performed using the QIAamp DNA stool Mini kit (Qiagen, Hilden, Germany) following the manufacturer's instructions. DNA concentration was determined by absorbance at $260 \mathrm{~nm}$ (A260), and purity was estimated by determining the A260/A280 ratio with a Nanodrop spectrophotometer (Nanodrop Technologies, Wilmington, DE, USA).

\section{PCR amplification and analysis of 165 rDNA sequences}

$16 \mathrm{~S}$ sequences are marker genes present in most microbial species but with sequence variations that allow for accurate separation between different taxonomical groups [14]. Variable regions 2-3 of the 16S rDNA gene were amplified using TaKaRa Ex Taq PCR mixture (TAKARA Bio USA, Madison, WI, USA) and the PCR primers HDA1 (5'-GACTCCTACGGGAGGCAGCAGT-3') and HDA2 (5'-GTATTACCGCGGCTGC TGGCAC-3'). Forward primers were designed with the adaptor A sequence (CGTATCGCCTCCCTCGC GCCA) plus a key sequence (TCAG) and reverse primers with the adaptor B sequence (CTATGCGCCTT GCCAGCCCG) plus a key sequence (TCGA). 454-adaptors (Roche Applied Science, Indianapolis, IN, USA) were included in the forward primer followed by a $10 \mathrm{bp}$ sample-specific multiplex identifier. The PCR programme was set as follows: $95^{\circ} \mathrm{C}$ for $10 \mathrm{~min}$ then 30 cycles at $95^{\circ} \mathrm{C}$ for $1 \mathrm{~min}, 50^{\circ} \mathrm{C}$ for $1 \mathrm{~min}$ and $72^{\circ} \mathrm{C}$ for $1.5 \mathrm{~min}$, followed by $72^{\circ} \mathrm{C}$ for $10 \mathrm{~min}$. After agarose gel electrophoresis, PCR products were purified twice using the Agencourt AMPure Kit (Beckman Coulter, Milan, Italy) and quantified using the Quant-iT PicoGreen dsDNA assay kit (Invitrogen, Burlington, ON, Canada). An equimolar pool was obtained prior to further processing and sequencing in a GS Junior 454 platform (Roche Applied Science) according to the manufacturer's protocols using a Titanium chemistry apparatus (Roche Applied Science).

\section{Bioinformatic analysis}

The 454 pyrosequencing datasets were analysed using the Quantitative Insights into Microbial Ecology (QIIME) 1.8.0 software (http://qiime.org). Raw reads were initially filtered according to the 454 amplicon processing pipeline. The pyrosequencing reads were de-multiplexed and further filtered through the split_library.py script of QIIME. In order to guarantee a higher level of accuracy, the reads were excluded from analysis if they had an average quality score $<25$ and if there were ambiguous base calls. The pipeline analysis used to analyse $16 \mathrm{~S}$ gene reads was as follows: sequences that passed the quality filter were denoised and singletons were excluded. Operational taxonomic units (OTUs) were picked by clustering sequences at a similarity of $>97 \%$ and the representative sequences, chosen as the most abundant in each cluster, were submitted to the UCLUST-consensus taxonomy assignment (http://drive5.com/usearch/ manual/uclust_algo.html) to obtain the taxonomy assignment and the relative abundance of each OTU using the Greengenes 16S rRNA gene database (http://greengenes.lbl.gov/cgi-bin/nph-index.cgi). $\alpha$ - and $\beta$-diversity were evaluated through QIIME as described previously [15]. The OTU table filtered at $1 \%$ 
abundance was used to generate an OTU network by QIIME and a bipartite graph was constructed in which each node represented either a sample or a bacterial OTU. Connections were drawn between samples and OTUs, with edge weights defined as the number of sequences from each OTU that occurred in each sample. Unweighted UniFrac distance matrix was used to perform ANOSIM statistical tests through the compare_categories.py script of QIIME. Distances between microbial communities from each sample were calculated with the jackknife coefficient. They were represented by unweighted pair group method with arithmetic mean algorithm (UPGMA) clustering trees describing the dissimilarity between multiple samples generated by QIIME.

\section{Statistical analysis}

Changes in body weight were analysed by two-way ANOVA considering time (before versus after the 6-week period) and treatment (intermittent hypoxia versus normoxia). Comparison of the relative abundances of the OTUs between groups was performed using a Wilcoxon test with a continuity correction using a software package, Explicet, specifically addressed to analyse microbiome data [16]. $\alpha$ - and $\beta$-diversities were achieved by QIIME: $\alpha$-diversity using a nonparametric $t$-test with a default number of 999 Monte Carlo permutations, and $\beta$-diversity using the ANOSIM statistical method with 99 permutations. A p-value $<0.05$ was designated as achieving statistical significance.

\section{Results}

\section{$\mathrm{PaO}_{2}$ measurement in the gut lumen}

Intermittent hypoxia exposures and the resultant episodic swings of oxygen saturation in arterial blood translated into events of hypoxia/re-oxygenation experienced by the microbiota closer to the gut epithelium. As anticipated, during normoxic breathing $\mathrm{PaO}_{2}$ within the faeces in the area close to the centre of the small intestine (i.e. far away from the epithelial capillaries) was virtually nil. As the oxymeter probe tip approached the contralateral wall of the bowel, $\mathrm{PaO}_{2}$ progressively increased to values of $\sim 50 \mathrm{mmHg}$, corresponding to those recorded in close vicinity of the bowel epithelium. Application of intermittent hypoxia resulted in obvious swings in $\mathrm{PaO}_{2}$ with oscillatory amplitudes that decreased as the microelectrode tip moved towards the centre of the faecal bolus in the range of $100-200 \mu \mathrm{m}$, as shown in figure 1. This figure includes the values of $\mathrm{PaO}_{2}$ measured in baseline conditions (animal breathing
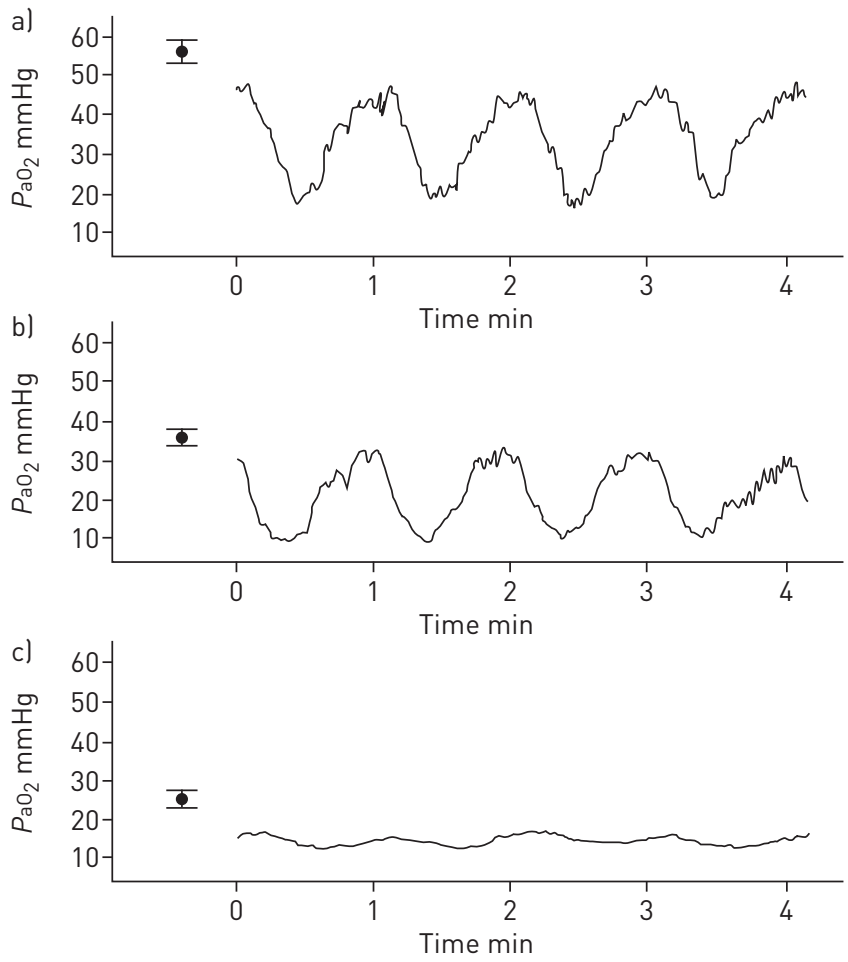

FIGURE 1 Examples of partial pressure of oxygen $\left(\mathrm{PaO}_{2}\right)$ within the small bowel. Time course of $\mathrm{PaO}_{2}$ recorded in the small bowel during application of intermittent hypoxia mimicking obstructive sleep apnoea at three points within the lumen. a) Immediate vicinity of the bowel epithelium, b) $100 \mu \mathrm{m}$ towards the central part of the bowel lumen and c) 200 $\mu \mathrm{m}$ towards the central part of the bowel lumen. For each point, the symbol represents the mean \pm SD of 2 -min baseline (normoxia) values measured prior the application of intermittent hypoxia. 
normoxic room air) just before application of intermittent hypoxia. These data illustrate that, as expected, under conditions of normoxia there is a gradient of oxygen in the faeces and when the animal is subjected to intermittent hypoxia, $\mathrm{PaO}_{2}$ experiences swing together with a reduction of mean level.

\section{Change in body weight}

Mean \pm SD baseline weight was $22.11 \pm 0.52 \mathrm{~g}$ and $22.71 \pm 0.30 \mathrm{~g}$ for the normoxia and intermittent hypoxia groups, respectively. After the 6-week period, weights were $24.44 \pm 0.67 \mathrm{~g}$ and $23.41 \pm 0.29 \mathrm{~g}$, respectively. Time was a significant factor $(\mathrm{p}<0.001)$ in weight gain whereas treatment (intermittent hypoxia versus normoxia) was not significant $(\mathrm{p}=0.747)$.

\section{Diversity estimation of gut microbiota associated with intermittent hypoxia and normoxia}

A total of 88884 16S rRNA gene sequences with an average of 7407 sequences per sample successfully passed the filters applied through QIIME. The faecal microbiota of all mice after QIIME was composed by 883 OTUs with a relative abundance $>1 \%$ in at least four samples. Prior to assessing $\alpha$ - and $\beta$-diversity measures, samples were rarefied to 2500 sequences, which corresponded to the lowest number of quality reads obtained from any individual sample in the dataset. As a result, two samples were eliminated from analysis in each study group due to their low quality level. The intermittent hypoxia group had a greater number of OTUs than the normoxia group (mean 284 versus 221, respectively; $\mathrm{p}=0.019$ ). The average diversity (Shannon) and community richness (Chao) of each group suggested a trend toward higher bacterial diversity and richness in stool samples from intermittent hypoxia-exposed mice compared to those from the control group (table 1). The rarefaction curve of observed OTUs calculated at 3\% distance started to plateau at $\sim 300$ reads, suggesting that a higher number of reads per sample would not have provided a more comprehensive catalogue of bacterial taxa. The Shannon index was significantly different between intermittent hypoxia and normoxia groups $(\mathrm{p}=0.010)$, indicating an increase in evenness of species distribution. In other words, the few dominant species decreased abundance in favour of several minority members of the community. However, the Chaol index of bacterial richness did not plateau with increasing reads, indicating that main components of community diversity would possibly not be detected with our level of depth. An unweighted Unifrac principal component analysis based on the relative abundance of OTUs for each sample provided useful information about the phylogenetic relationship among the faecal bacterial microbiota in both study groups. All samples from the normoxia group were separated from the $\mathrm{IH}$ group in the combinations of coordinates, as demonstrated by the two principal component scores that accounted for 59\% (PC1) and 17\% (PC2) of total variance. ANOSIM with permutations confirmed significant separation of groups $(\mathrm{p}=0.01)$ (fig. 2), indicating that there are clear differences in the microbial composition of intermittent hypoxia and normoxia groups. The UPGMA tree represented the similarity of microbial community membership of intermittent hypoxia and normoxia mice. Remarkably, intermittent hypoxia and normoxia mice clustered separately from each other, and there was a clear distinction (high (75-100\%) jackknife support) among the samples from the intermittent and normoxia groups (fig. 3).

\section{S rRNA gene pyrosequencing of intermittent hypoxia and normoxia in faecal samples from mice}

In this study, there were significant variations in the composition of faecal microbiota among the intermittent hypoxia and normoxia groups at different bacterial levels. At the phylum level, the majority of the OTUs belonged to Bacteroidetes (69.79\% intermittent hypoxia versus $73.61 \%$ normoxia; $\mathrm{p}=0.10$ ). Firmicutes was the next most abundant $(22.74 \%$ intermittent hypoxia versus $27.63 \%$ normoxia; $\mathrm{p}=0.49)$ followed by Proteobacterias $(1.20 \%$ intermittent hypoxia versus $1.50 \%$ normoxia; $\mathrm{p}=0.71)$. The remainder

TABLE 1 Estimates of microbial richness and diversity indices among microbial communities obtained from faecal samples of intermittent hypoxia- and normoxia-exposed mice

\begin{tabular}{lccc} 
& Intermittent hypoxia & Normoxia & p-value* \\
\hline Subjects $\mathbf{n}$ & 10 & 10 & \\
Chao 1 & $371.75 \pm 29.52$ & $325.28 \pm 64.44$ & 0.1 \\
Shannon & $5.66 \pm 0.14$ & $5.39 \pm 0.21$ & 0.001 \\
\hline
\end{tabular}

Data are presented as mean $\pm S D$, unless otherwise stated. The microbial richness estimator (Chao 1) and the diversity indices estimator (Shannon) were calculated at $3 \%$ distance. *: values are statistically significant for $p<0.05$. 


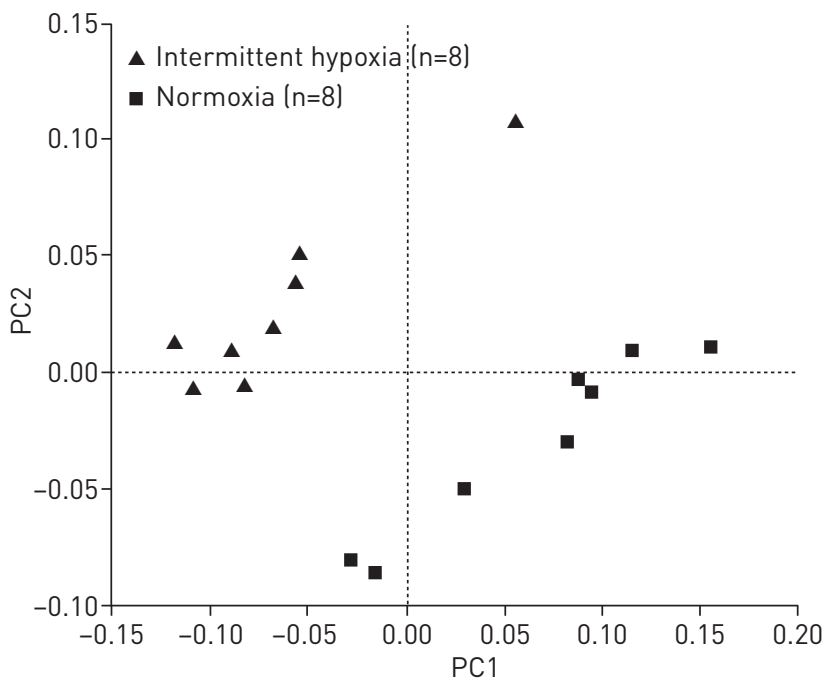

FIGURE 2 Principal component analysis, PC1 versus PC2, of bacterial communities from faecal samples subjected to 454 sequencing. Positions of the bacterial communities for each species along the two first principal coordinate axes are illustrated, along with the percentage of variation explained by each axis. Results are based on unweighted Unifrac metrics. $\mathrm{PC} 1=59.11 \%$; $\mathrm{PC} 2=17.39 \%$.

of the bacterial population belonged to seven other phyla that had a relative abundance $<1 \%$ in at least four samples (fig. 4). None of the phyla exhibited the presence of statistically significant differences between the two study groups. In addition, the Firmicutes/Bacteroidetes ratio was not significantly different among the control and the intermittent hypoxia group ( $\mathrm{p}=0.10)$.

27 families were detected among all mice groups with the most dominant being S24-7 (47.70\% intermittent hypoxia versus $53.21 \%$ normoxia; $\mathrm{p}=0.12$ ), followed by Alcaligenaceae (33.33\% intermittent hypoxia versus $43.14 \%$ normoxia; $\mathrm{p}=0.27$ ), Desulfovibrionaceae ( $25.31 \%$ intermittent hypoxia versus $5.01 \%$ normoxia; $\mathrm{p}=0.27$ ) and Ruminococcaceae $(20.74 \%$ intermittent hypoxia versus $30.29 \%$ normoxia; $\mathrm{p}=0.37)$. However, we found significant differences between nine other minority families. The relative abundances of Prevotellaceae ( $11.82 \%$ intermittent hypoxia versus $5.94 \%$ normoxia; $\mathrm{p}=0.01)$, Paraprevotellaceae $(7.64 \%$

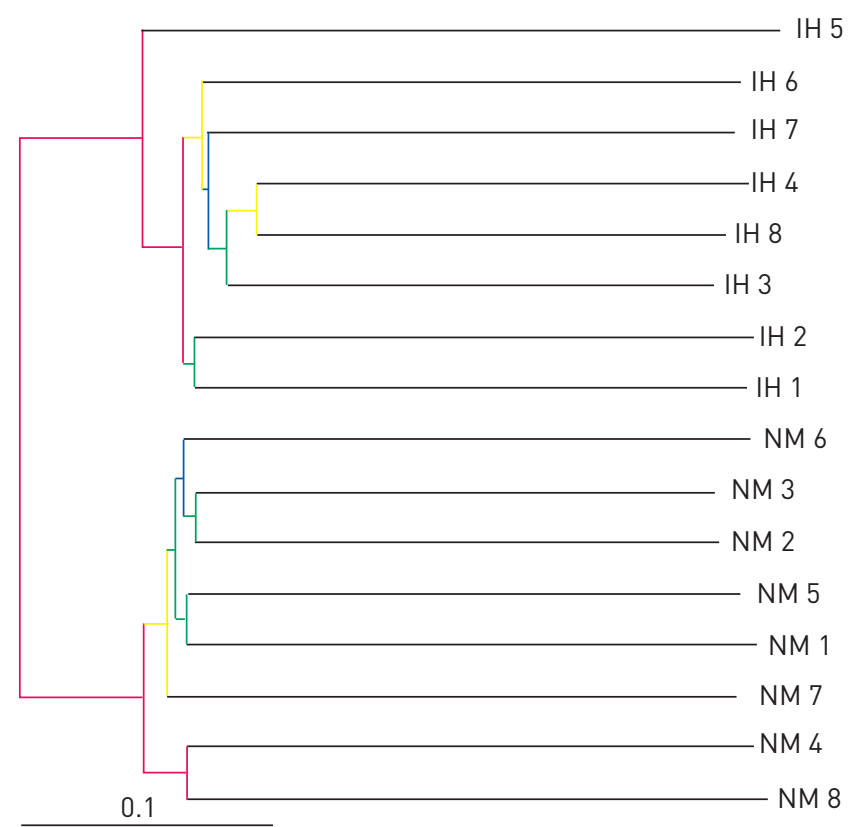

FIGURE 3 Unweighted pair group method with arithmetic mean algorithm hierarchical clustering using the unweighted Unifrac distance matrix. The colours represent different jackknife support. Red: 75-100\% support; yellow: 50-75\% support; green: $25-50 \%$ support; blue: <25\% support. The bar represents community dissimilarity. IH: intermittent hypoxia; NM: normoxia. 


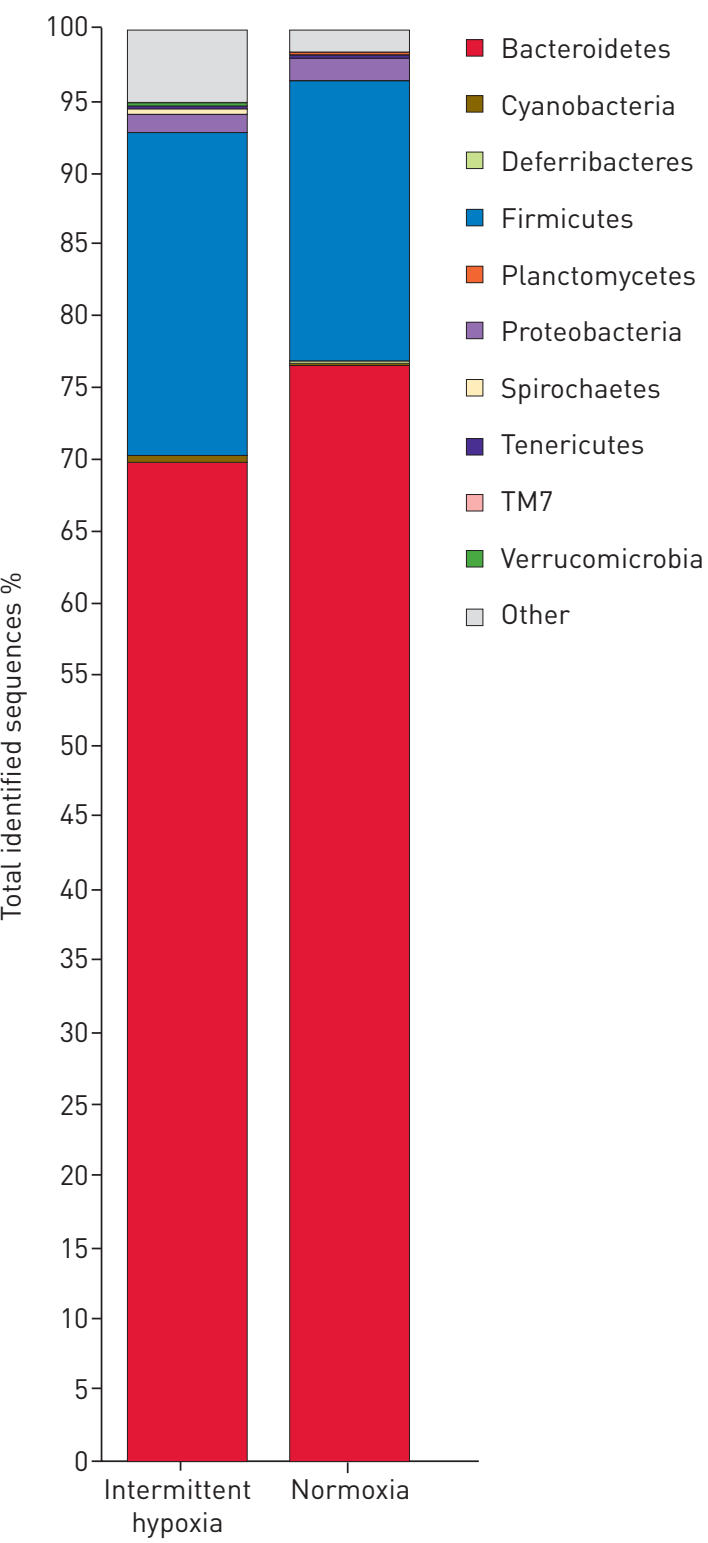

FIGURE 4 Pyrosequencing analysis of phyla in the intermittent hypoxia and normoxia groups of mice.

intermittent hypoxia versus $3.78 \%$ normoxia; $\mathrm{p}=0.03)$ and Lachnospiraceae $(6.03 \%$ intermittent hypoxia versus $1.77 \%$ normoxia; $\mathrm{p}<0.001)$ were significantly higher in the intermittent hypoxia group compared to the normoxia group. There was a significantly lower level of Erysipelotrichaceae $(4.23 \%$ intermittent hypoxia versus $18.19 \%$ normoxia; $\mathrm{p}<0.001)$, Bacteroidaceae $(2.10 \%$ intermittent hypoxia versus $8.31 \%$ normoxia; $\mathrm{p}<0.01)$, Turicibacteraceae $(0.33 \%$ intermittent hypoxia versus $7.26 \%$ normoxia; $\mathrm{p}<0.001)$, Odoribacteraceae (1.34\% intermittent hypoxia versus $2.34 \%$ normoxia; $\mathrm{p}=0.04)$ and Peptococcaceae $(0.26 \%$ intermittent hypoxia versus $1.96 \%$ normoxia; $\mathrm{p}=0.01$ ) in the intermittent hypoxia group with respect to the normoxia group. Moreover, no significant differences between both study groups were found in other abundant families, including Porphyromonadaceae (1.66\% intermittent hypoxia versus $2.01 \%$ normoxia; $\mathrm{p}=0.27)$, Rikenellaceae $(6.29 \%$ intermittent hypoxia versus $7.95 \%$ normoxia; $p=0.08)$, Lactobacillaceae ( $2.39 \%$ intermittent hypoxia versus $2.45 \%$ normoxia; $p=0.20$ ), and Enterobacteriaceae (1.68 intermittent hypoxia versus $1.67 \%$ normoxia; $\mathrm{p}=0.65$ ) (fig. 5).

We also found significant differences in the microbial composition at the genus level between the study groups. A total of 23 genera were identified among the 20 sampled mice, with six significantly different genera between the intermittent hypoxia and the normoxia groups. Odoribacter $(1.34 \%$ intermittent hypoxia versus $2.34 \%$ normoxia; $\mathrm{p}=0.04)$, Bacteroides $(2.10 \%$ intermittent hypoxia versus $8.31 \%$ normoxia; $\mathrm{p}<0.001)$, Turicibacter $(1.33 \%$ intermittent hypoxia versus $7.26 \%$ normoxia; $\mathrm{p}<0.001)$ and Allobaculum 

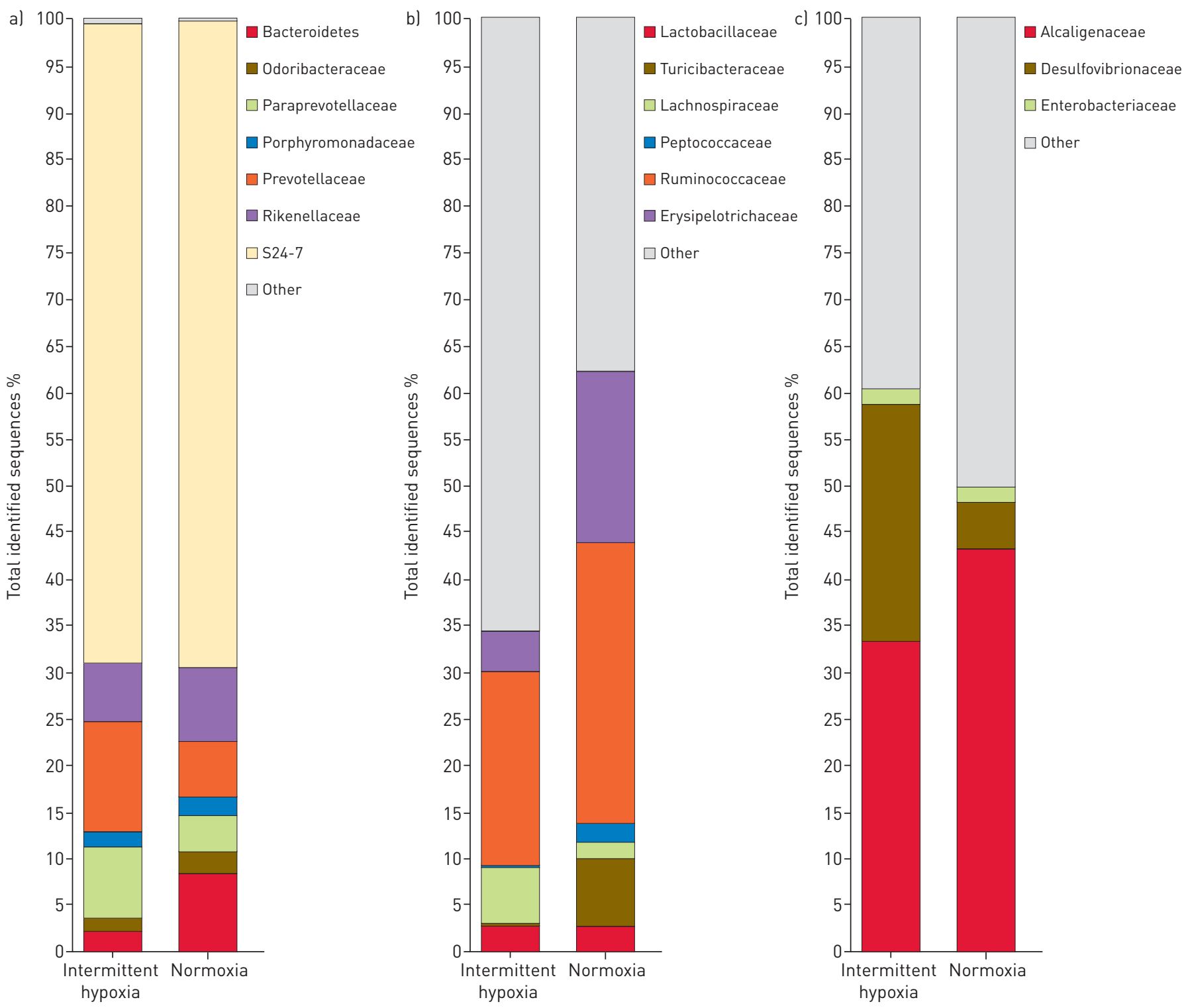

FIGURE 5 Family level microbial classification of bacteria from intermittent hypoxia and normoxia faecal samples. a) Bacteroidetes family, b) Firmicutes family and c) Proteobacteria family.

( $4.03 \%$ intermittent hypoxia versus $19.97 \%$ normoxia; $\mathrm{p}<0.001$ ), which constitute over $1 \%$ of the total bacteria in the faecal samples, were significantly more abundant in the normoxia group. Moreover, Paraprevotella (7.64\% intermittent hypoxia versus 3.69\% normoxia; $\mathrm{p}=0.03)$ and Prevotella $(11.82 \%$ intermittent hypoxia versus $5.94 \%$ normoxia; $\mathrm{p}=0.01$ ) exhibited a significantly higher abundance in the intermittent hypoxia group compared to the normoxia group. On the other hand, Desulfovibrio (24.89\% intermittent hypoxia versus $4.34 \%$ normoxia) was the most abundant genus in the intermittent hypoxia group, while the Sutterella (33.33\% intermittent hypoxia versus $43.14 \%$ normoxia) and Oscillospira (12.76\% intermittent hypoxia versus $25.09 \%$ normoxia) genera dominated the normoxia group. Differences in abundance for the top three genera between intermittent hypoxia and normoxia groups were not statistically significant ( $p>0.05$ in all cases) (fig. 6).

\section{Discussion}

To our knowledge, this is the first study examining the effects of intermittent hypoxia mimicking OSA on the composition of gut microbiome. Here, we have combined the measurement of oxygen concentration inside the gut (in the small bowel faeces) with community level taxonomic profiling of faecal microbiome to determining how the potential oscillations in oxygen tension impact on the host's gut microbiota. The pyrosequencing analysis of the $16 \mathrm{~S}$ rDNA gene-tags, applied to faecal samples from intermittent hypoxia 

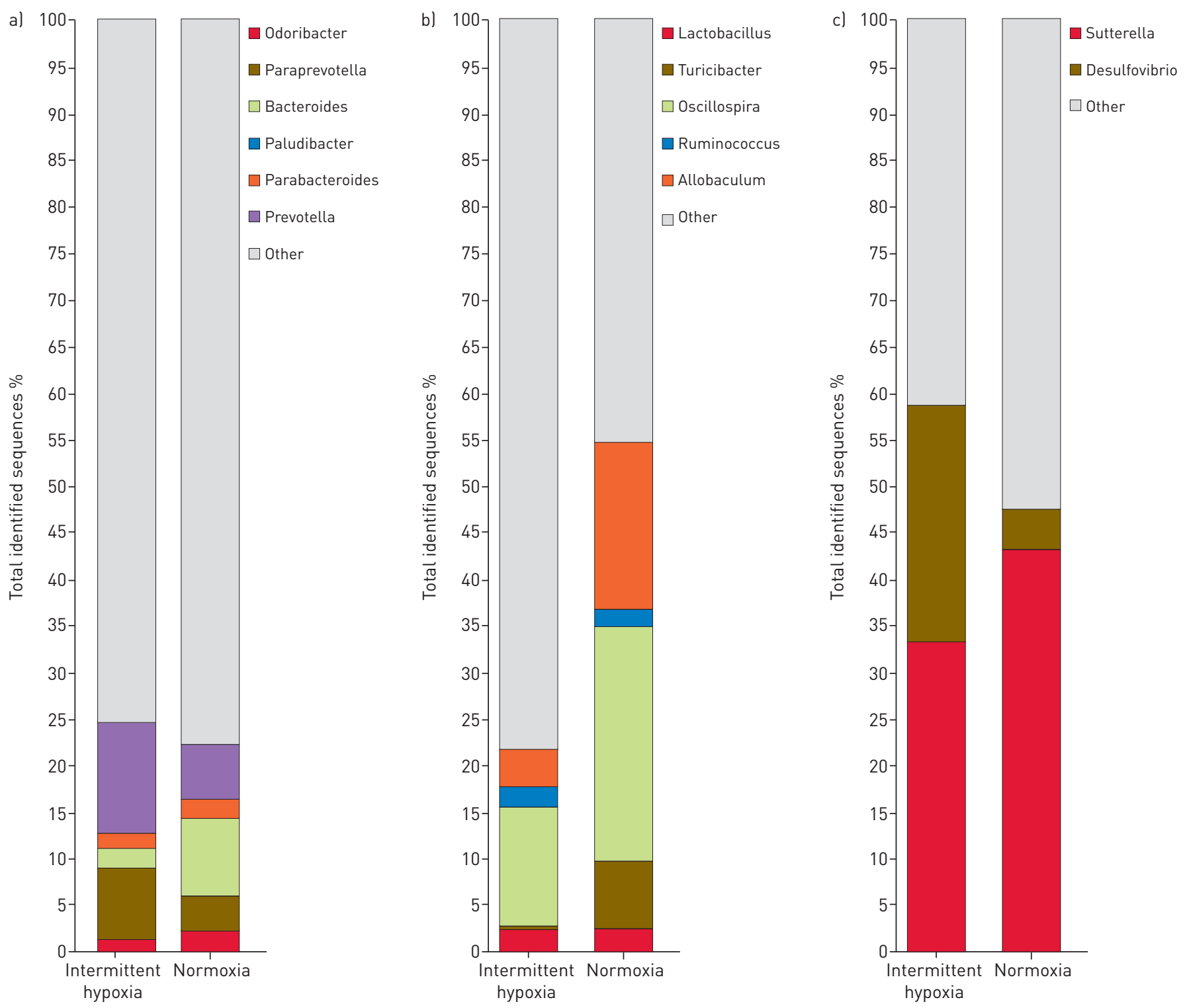

FIGURE 6 Relative abundance of bacterial genera in the microbiota of the intermittent hypoxia- and normoxia-exposed mice. a) Bacteroidetes genera, b) Firmicutes genera and c) Proteobacteria genera.

and normoxia mice, revealed large differences in the relative abundances of predominant taxa of phyla, family and genera levels.

Assessment of the changes in $\mathrm{PaO}_{2}$ induced by intermittent hypoxia exposure in the bowel was carried out with an oxygen sensor that was previously used to measure $\mathrm{PaO}_{2}$ within different tissues in animal models of OSA $[4,6]$. As expected, the level of oxygenation in the faeces decreased as the sensor approached the anoxic core of the intestinal content. However, it is remarkable that the $\mathrm{PaO}_{2}$ oscillations were detectable within a range of $\sim 0-200 \mu \mathrm{m}$ from the intestinal epithelial surface, demonstrating that relevant portions of microbiota are susceptible to alterations in oxygenation caused by intermittent hypoxia. These experimental data are consistent with theoretical predictions from the solution of the Fick's second law of diffusion for a semi-infinite medium (modelling free diffusion within the faeces from the epithelial surface). Indeed, the amplitude (A) of oxygen concentration oscillation at a distance $\mathrm{z}$ from the surface is: $A(z)=A_{0} \cdot e^{-\pi \cdot f \cdot z / D}$, where $f$ is the oscillation frequency and $D$ the coefficient of diffusion of oxygen in the medium. Considering that $D$ in the faeces is $2 \times 10^{-5} \mathrm{~cm}^{2} \cdot \mathrm{s}^{-1}$, a value similar to that of water and other biological tissues [17], and that the frequency of apnoeas is 60 events. $\mathrm{h}^{-1}$, it results that $\mathrm{A}(\mathrm{z}) / \mathrm{A}_{0=} \mathrm{e}^{-\mathrm{z} /(195 \mu \mathrm{m})}$ indicating that at 100,200 and $300 \mu \mathrm{m}$ from the epithelial surface the amplitude of $\mathrm{PaO}_{2}$ oscillations are reduced from $100 \%$ to $60 \%, 36 \%$ and $21 \%$, respectively. Although this theoretical prediction does not take into account potential oxygen consumption by aerobic microbia, 
it allows us to interpret the measured data, clearly indicating that intermittent hypoxia mimicking OSA is actually translated into environmental changes within the bowel and potentially sensed by the bowel microbiota.

Contrary to previous observations in conditions such as obesity, type 1 and 2 diabetes or Crohn's disease [18-21], we did not find significant differences in gut microbiota at the phylum level when comparing intermittent hypoxia and normoxia groups. By contrast, we found significant differences at the level of dominant microbiota families and genera: Prevotella, Paraprevotella, Desulfovibrio and Lachnospiraceae increased, whereas Bacteroides, Odoribacter, Turicibacter, Peptococcaceae and Erysipelotrichaceae decreased in the faeces of the intermittent hypoxia group. These findings suggest that the dominant microbiota families and genera are different after intermittent hypoxia exposures, with a relative enrichment in obligate anaerobes in the intermittent hypoxia group. The overall reduction in oxygen content inside the gut when the animal is under intermittent hypoxia compared with normoxia (fig. 1) would confer an ecological selective advantage to obligate anaerobes, allowing them to be more competitive and enabling them to overgrow. Conversely, the facultative anaerobes or even aerobes would be at a disadvantage. It should be mentioned the analysed faecal samples not only come from the area close to the intestine epithelium where hypoxia/re-oxygenation is most pronounced, but also from the inner regions of the intestinal lumen, which has a more continuous pattern of very severe hypoxia.

Recently, three robust clusters referred to as "enterotypes", which are not country or continent specific, have been identified. Assignment of an individual microbiome into a given enterotype is based upon its relative enrichment into one of three genera: Bacteroides (enterotype 1), Prevotella (enterotype 2) or Ruminococcus (enterotype 3) [22]. The normoxia and intermittent hypoxia groups could be classified into entrerotypes 1 and 2 given their relative enrichment in Bacteroides and Prevotella, respectively, and the significant increase in the abundance of Desulfovibrio in the intermittent hypoxia microbiomes [22]. The co-occurrence of Prevotella and Desulfovibrio found in the intermittent hypoxia group suggests evolution of a mucin-degrading niche whereby the sulfate liberated during Prevotella-mediated mucin degradation is removed by Desulfovibrio, a process required to prevent sulfate inhibition of mucin degradation [22, 23]. Mucin degradation by bacteria is often regarded as an initial stage in pathogenesis, since it would disturb the protection of the host mucosal surfaces. In our study the significant increase in the abundance of Prevotella and Desulfovibrio (mucus-degrading microbes) in the intermittent hypoxia group may indicate a lack of this mucin on the epithelial layer of the gut, which could potentially lead to a significant alteration in intestinal permeability. Furthermore, the enriched bacteria Desulfovibrio reduces sulfate in order to produce hydrogen sulfide, which has been reported as a possible contributing risk factor of colorectal cancer $[24,25]$.

Several members of the obligate anaerobic Lachnospiraceae family were significantly enriched in the intermittent hypoxia mice and have been previously linked to obesity [26] and the ability to produce butyric acid [27], a substance that is important for both microbial and host epithelial cell growth. In addition, our results also indicate that mice under intermittent hypoxia have increased bacterial diversity and cluster separately when compared to normoxia controls, as is clearly indicated by the principal coordinate plot and UPGMA dendrograms. The increase in the diversity of gut bacteria in intermittent hypoxia, as indicated by Shannon diversity index, may allow improved microbial integrity and increase the ability to protect the human intestine from environmental stresses, such as changes in the oxygen level inside the gut. Although the intestinal epithelium is remarkably resistant to hypoxia, absorptive and barrier epithelial functions are regulated by oxygen [28]. In fact, hypoxia/re-oxygenation can directly impair cellular function via increases in permeability and bacterial translocation and decreases in tight junction integrity [29]. Recently, KHEIRANDISH-GozAL et al. [30] have demonstrated that children with OSA, even those who are not obese, exhibit significantly higher lipopolysaccharide binding protein plasma levels when compared with healthy controls. These results are supportive of the possibility that alterations in the gut microbiota may promote changes in intestinal permeability or, alternatively, induce microbial translocation, all of which could lead to increased levels of systemic lipopolysaccharide. The increased circulating endotoxin levels would then promote innate immunity events triggering low-grade systemic inflammatory processes, which may result in metabolic dysfunction.

In summary, although it cannot be ruled out that the alterations in microbiota observed in the intermittent hypoxia mice could be due, in part, to systemic immune pathways triggered by intermittent hypoxia in the host that are capable of modifying the microbiota, it is reasonable to assume that the actual reported changes of $\mathrm{PaO}_{2}$ inside the gut are driving the changes in the abundance of obligate anaerobes and the increase in diversity observed in the bacterial communities of faecal microbiota of mice exposed to realistic simulation of the intermittent hypoxemia that characterises OSA. The hypothesis that intermittent hypoxia has a significant impact on the overall microbial community structure of mice was confirmed, suggesting that the homeostatic relationships between host and gut microbiota could be compromised 
in OSA patients. This study opens a new area of research in the field of sleep apnoea, and definitely warrants further studies on the effects of intermittent hypoxia, as well as sleep fragmentation [31], on gut microbiota and its modulation of host metabolism in both animal models and patients.

\section{References}

1 Peppard PE, Young T, Barnet $\mathrm{JH}$, et al. Increased prevalence of sleep-disordered breathing in adults. Am J Epidemiol 2013; 177: 1006-1014.

2 Lam JC, Mak JC, Ip MS. Obesity, obstructive sleep apnoea and metabolic syndrome. Respirology 2012; 17: 223-236.

3 Lavie L. Oxidative stress in obstructive sleep apnea and intermittent hypoxia - Revisited - The bad ugly and good: Implications to the heart and brain. Sleep Med Rev 2014; 20C: 27-45.

4 Almendros I, Montserrat JM, Torres M, et al. Changes in oxygen partial pressure of brain tissue in an animal model of obstructive apnea. Respir Res 2010; 11: 3.

5 Reinke C, Bevans-Fonti S, Drager LF, et al. Effects of different acute hypoxic regimens on tissue oxygen profiles and metabolic outcomes. J Appl Physiol 2011; 111: 881-890.

6 Torres M, Laguna-Barraza R, Dalmases M, et al. Male fertility is reduced by chronic intermittent hypoxia mimicking sleep apnea in mice. Sleep 2014; 37: 1757-1765.

7 Qin J, Li R, Raes J, et al. A human gut microbial gene catalogue established by metagenomic sequencing. Nature 2010; 464: 59-65.

8 Espey MG. Role of oxygen gradients in shaping redox relationships between the human intestine and its microbiota. Free Radic Biol Med 2013; 55: 130-140.

9 Albenberg L, Esipova TV, Judge CP, et al. Correlation between intraluminal oxygen gradient and radial partitioning of intestinal microbiota. Gastroenterology 2014; 147: 1055-1063.

10 Le Chatelier E, Nielsen T, Qin J, et al. Richness of human gut microbiome correlates with metabolic markers. Nature 2013; 500: 541-546.

11 Moreno-Indias I, Cardona F, Tinahones FJ, et al. Impact of the gut microbiota on the development of obesity and type 2 diabetes mellitus. Front Microbiol 2014; 5: 190.

12 Bonsignore MR, Esquinas C, Barceló A, et al. Metabolic syndrome, insulin resistance and sleepiness in real-life obstructive sleep apnoea. Eur Respir J 2012; 39: 1136-1143.

13 Ong CW, O'Driscoll DM, Truby H, et al. The reciprocal interaction between obesity and obstructive sleep apnoea. Sleep Med Rev 2013; 17: 123-131.

14 Chakravorty S, Helb D, Burday M, et al. A detailed analysis of 16S ribosomal RNA gene segments for the diagnosis of pathogenic bacteria. J Microbiol Methods 2007; 69: 330-339.

15 De Filippis F, La Storia A, Villani F, et al. Exploring the sources of bacterial spoilers in beefsteaks by culture-independent high-throughput sequencing. PLoS One 2013; 8: e70222.

16 Robertson CE, Harris JK, Wagner BD, et al. Explicet: graphical user interface software for metadata-driven management, analysis, and visualization of microbiome data. Bioinformatics 2013; 29: 3100-3101.

17 Colom A, Galgoczy R, Almendros I, et al. Oxygen diffusion and consumption in extracellular matrix gels: implications for designing three-dimensional cultures. J Biomed Mater Res A 2014; 102: 2776-2784.

18 Murri M, Leiva I, Gomez-Zumaquero JM, et al. Gut microbiota in children with type 1 diabetes differs from that in healthy children: a case-control study. BMC Med 2013; 11: 46.

19 Ley RE. Obesity and the human microbiome. Curr Opin Gastroenterol 2010; 26: 5-11.

20 Willing B, Halfvarson J, Dicksved J, et al. Twin studies reveal specific imbalances in the mucosa-associated microbiota of patients with ileal Crohn's disease. Inflamm Bowel Dis 2009; 15: 653-660.

21 Larsen N, Vogensen FK, van den Berg FW, et al. Gut microbiota in human adults with type 2 diabetes differs from non-diabetic adults. PLoS One 2010; 5: e9085.

22 Arumugam M, Raes J, Pelletier E, et al. Enterotypes of the human gut microbiome. Nature 2011; 473: 174-180.

23 Payne AN, Chassard C, Lacroix C. Gut microbial adaptation to dietary consumption of fructose, artificial sweeteners and sugar alcohols: implications for host-microbe interactions contributing to obesity. Obes Rev 2012; 13: 799-809.

24 Huycke MM, Gaskins HR. Commensal bacteria, redox stress, and colorectal cancer: mechanisms and models. Exp Biol Med (Maywood) 2004; 229: 586-597.

25 Muyzer G, Stams AJ. The ecology and biotechnology of sulphate-reducing bacteria. Nat Rev Microbiol 2008; 6: 441-454.

26 Cho I, Yamanishi S, Cox L, et al. Antibiotics in early life alter the murine colonic microbiome and adiposity. Nature 2012; 488: 621-626.

27 Duncan SH, Barcenilla A, Stewart CS, et al. Acetate utilization and butyryl coenzyme A (CoA): acetate-CoA transferase in butyrate-producing bacteria from the human large intestine. Appl Environ Microbiol 2002; 68: 5186-5190.

28 Taylor CT, Colgan SP. Hypoxia and gastrointestinal disease. J Mol Med (Berl) 2007; 85: 1295-1300.

29 Xu DZ, Lu Q, Kubicka R, et al. The effect of hypoxia/reoxygenation on the cellular function of intestinal epithelial cells. J Trauma 1999; 46: 280-285.

30 Kheirandish-Gozal L, Peris E, Wang Y, et al. Lipopolysaccharide-binding protein plasma levels in children: effects of obstructive sleep apnea and obesity. J Clin Endocrinol Metab 2014; 99: 656-663.

31 Carreras A, Leone V, Peris E, et al. Sleep fragmentation alters gut microbiota: potential association with metabolic alterations in mice. Am J Respir Crit Care Med 2014; 189: A2414. 